\title{
A Case of Recurrent Metastatic Parotid Acinic Cell Carcinoma Responsive to Pembrolizumab
}

\author{
GAI YAMASHITA, ISAKU OKAMOTO, AKIRA SHIMIZU, KUNIHIKO TOKASHIKI, \\ TAKURO OKADA, HIROKI SATO and KIYOAKI TSUKAHARA
}

Department of Otorhinolaryngology, Head and Neck Surgery, Tokyo Medical University, Tokyo, Japan

\begin{abstract}
Background: No clear chemotherapy regimen for recurrent or metastatic parotid cancer exists. We describe our experience with pembrolizumab to treat recurrent or metastatic parotid cancer. Case Report: A 73-year-old woman with swelling in the lower part of the right ear for 10 years before surgery was diagnosed with right parotid cancer, underwent total right parotidectomy, and reported recurrence. She requested treatment due to diminished quality of life caused by neurological symptoms. Tissue was collected from the recurrent lesion and its combined positive score was $>20$; pembrolizumab was started 9 years postoperatively. Results: To date, the patient has received 14 cycles of pembrolizumab. Evaluation by computed tomography showed a partial response to treatment. The only immune-related adverse event was grade 1 pneumonia in both lungs. Conclusion: Significant response to pembrolizumab in recurrent or metastatic parotid cancer is rarely reported, making this a remarkable case. We plan to continue pembrolizumab administration.
\end{abstract}

Salivary gland cancer, including parotid cancer, is a relatively rare tumour, accounting for $<5 \%$ of all head and neck cancers (1), of which there are 23 organisational types (2). There are no established chemotherapy regimen guidelines for recurrent or metastatic parotid cancer from the National Comprehensive Cancer Network (3). Existing recommendations include cisplatin/vinorelbine and cisplatin/doxorubicin/cyclophosphamide as category $2 \mathrm{~B}$

This article is freely accessible online.

Correspondence to: Isaku Okamoto, MD, Ph.D., Department of Otorhinolaryngology, Head and Neck Surgery, Tokyo Medical University, 6-7-1 Nishishinjuku, Shinjuku-ku, Tokyo 160-0023, Japan. Tel.: +81 333426111; Fax: +81 333469275, e-mail: isaku@tokyo-med.ac.jp

Key Words: Parotid cancer, acinic cell carcinoma, head and neck cancer, pembrolizumab. regimens. KEYNOTE-048, a randomised phase III clinical trial involving participants with untreated, locally recurrent, or metastatic head and neck squamous cell carcinoma, showed that the single-agent immune checkpoint inhibitor (ICI) pembrolizumab prolonged overall survival (OS) compared with conventional standard therapy in patients with recurrent metastatic squamous cell carcinoma (SCC) of the head and neck with a combined positive score (CPS) $>1$ and CPS $>20$. The CPS is given by summing the number of programmed cell death ligand 1(PD-L1)-stained cells (tumor cells, lymphocytes, macrophages) and dividing the result by the total number of viable tumor cells, multiplied by 100 (4). Pembrolizumab was approved for insurance coverage in Japan in December 2019 for recurrent or metastatic head and neck cancer; parotid cancer is considered a type of head and neck cancer that can be treated with pembrolizumab in Japan.

A case report documented the effectiveness of pembrolizumab in the treatment of an undifferentiated parotid cancer; however, this has not been described yet for acinic cell carcinoma (5). In addition, Okamoto et al. (6) reported that nivolumab, an ICI, significantly prolonged OS in patients with recurrent and metastatic head and neck cancer, including SCC and non-SCC, when PD-L1 expression was $\geq 40 \%$. However, pembrolizumab has not yet been shown to be effective in the treatment of non-SCC recurrent metastatic head and neck cancer.

Our patient's case is important because pembrolizumab is rarely administered for salivary gland cancer. In this report, we describe our experience with pembrolizumab for the treatment of recurrent or metastatic parotid cancer.

\section{Case Report}

A 73-year-old woman presented with the chief complaint of swelling of the right parotid region. Her medical history included surgery for oral cancer at the age of 48 years, smoking five cigarettes per day from 20 to 35 years old, but no history of alcohol consumption. There was no family history of parotid disease. The patient's current medical history included swelling in the lower part of the right ear 


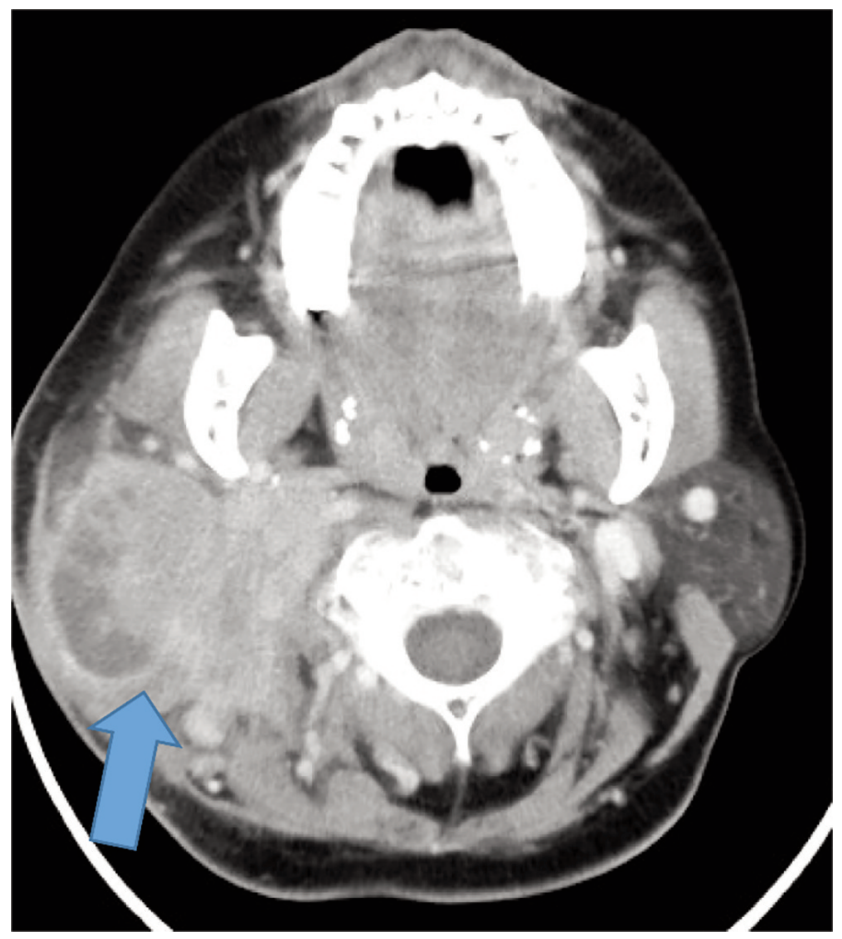

Figure 1. Preoperative findings in a 73-year-old woman presenting with swelling in the right parotid region. Cervical contrast-enhanced computed tomography scan (axial section). An irregular tumour (blue arrow) about $70 \mathrm{~mm}$ in length and diameter can be seen in the right parotid gland.

that had begun 10 years before total parotidectomy and gradually worsened. She was diagnosed with acinic cell carcinoma after needle biopsy at a local general hospital. The patient was then referred to our hospital. Initial examination revealed a smooth mass measuring $91 \mathrm{~mm} \times 68 \mathrm{~mm}$ in the lower part of the right ear. No facial nerve palsy was observed. Intraoral findings showed a surgical scar on the anterior palatal arch, but no other abnormal findings in the oral cavity, pharynx, or larynx.

Imaging findings at the initial examination included cervicothoracic contrast-enhanced computed tomography (CT) (Figure 1) showing an irregular tumour that was $70 \mathrm{~mm}$ in length and diameter in the right parotid gland. The tumour was observed to be a single mass surrounded by lymph nodes. However, there were no lymph node metastases or significant findings at other sites.

Fluorodeoxyglucose positron-emission tomography/CT revealed a strong accumulation (maximum standardised uptake value of 7.86) in the right parotid tumour. It was difficult to clearly separate the tumour from the surrounding lymph nodes; however, there were no other findings suggestive of metastasis. The patient was diagnosed with right parotid cancer (acinic cell carcinoma) cT3N2bM0 according to the Union for International Cancer Control version 7 criteria (7).

The patient underwent total right parotidectomy with excision of the mandibular branch of the facial nerve, and right neck dissection (IIa+IIb). In addition, dynamic reconstruction using the great auricular nerve and static reconstruction using the femoral fascia were performed to prevent facial nerve palsy.

Subsequently, the patient was regularly followed-up by her local doctor. Cervicothoracic contrast-enhanced CT at 4 years after surgery revealed a recurrent lesion from the deep parotid gland to the parapharyngeal space. Surgery, radiation therapy, and heavy-particle therapy were presented as treatment options; however, the patient refused them and remained under observation.

Eventually, on regular follow-up imaging, the lesion in the lower right ear appeared to increase in size. Cervicothoracic CT at 9 years postoperatively showed that the tumour had invaded the right jugular foramen and hypoglossal neural tube (Figure 2A). The lesion in the jugular foramen extended intracranially along the right internal jugular vein (Figure 2B). The patient had a new jugular foramen syndrome with right facial nerve palsy, hoarseness, dysphagia, and curtain signs, as well as headache due to intracranial tumour extension.

Fluorodeoxyglucose positron-emission tomography (Figure 2C and D) showed accumulation from the right parotid to the lateral pharyngeal lymph node and right supraclavicular lymph node. With symptom appearance, the patient's quality of life decreased significantly, and she requested treatment.

Because pembrolizumab was approved for the treatment of recurrent and metastatic head and neck cancer, we offered it to the patient, to which she agreed. A biopsy performed at the recurrence site confirmed acinic cell carcinoma. A CPS $>20$ was obtained. These results were promising for the efficacy of pembrolizumab therapy. Thus, 9 years after surgery, the patient was started on pembrolizumab at $200 \mathrm{mg}$ per dose every 3 weeks.

The findings prior to pembrolizumab administration showed that the tumour in the lower right ear was selfdestructive (Figure 2E) and right facial nerve palsy was present. Laryngoscopy revealed right recurrent nerve palsy, right curtain signs, and right hypoglossal nerve palsy.

Cervicothoracic contrast-enhanced CT (Figure 3A) to determine treatment effects after five cycles of pembrolizumab showed a marked reduction in the size of the mass that was bulging from the skin. The right supraclavicular lymph node metastasis also decreased in size, and the lymphatic portal was confirmed. Skin findings also showed tumour shrinkage in the lower part of the right ear (Figure 3B). Evaluation according to the Response Evaluation Criteria in Solid Tumors version 1.1 (8) showed a partial response (PR). In terms of safety, the only immune- 

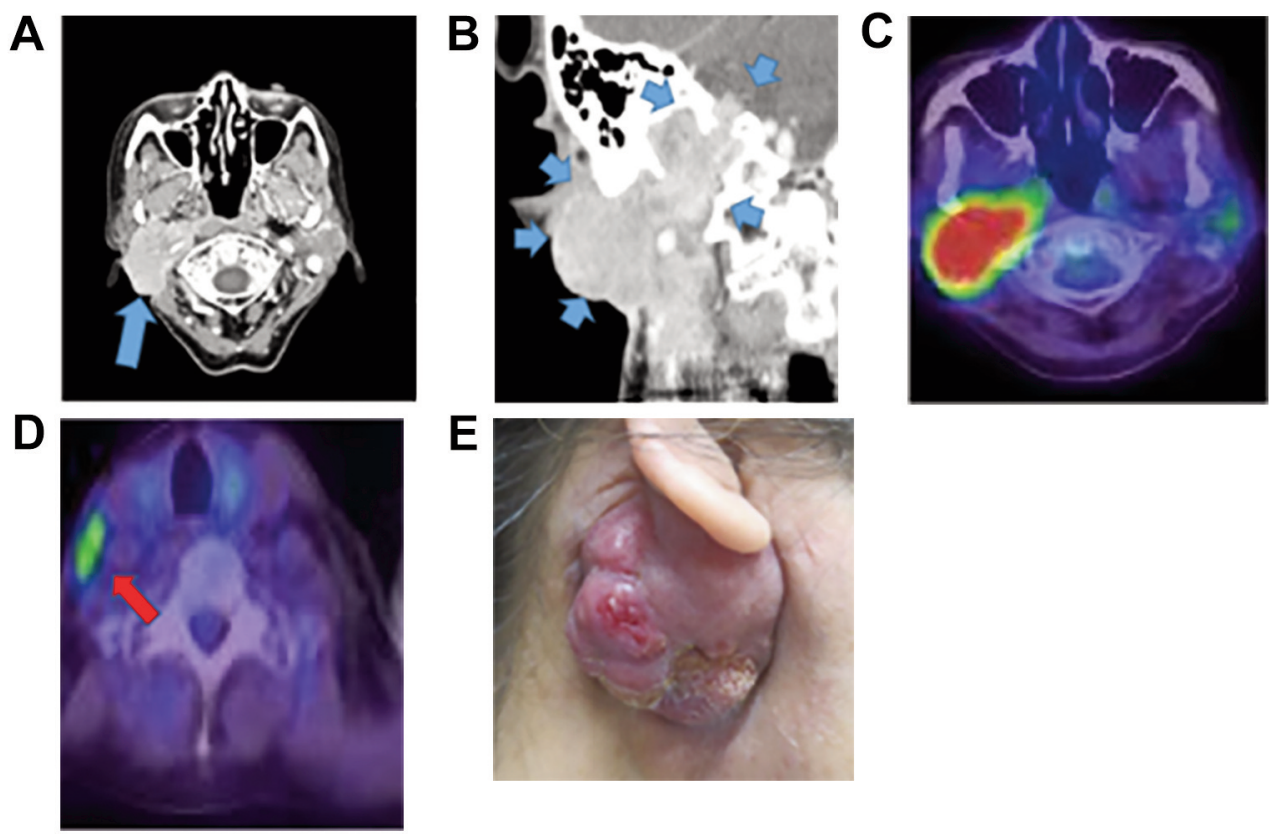

Figure 2. Findings following total right parotidectomy, pre-pembrolizumab treatment. A: Cervical contrast-enhanced computed tomography (CT) scan (axial section). Invasion of the right jugular foramen and hypoglossal neural tube by the recurrent lesion (blue arrow) can be seen. B: Cervical contrast-enhanced CT scan (coronal section). The recurrent lesion (blue arrow) extends intracranially along the right internal jugular vein. C: Fluorodeoxyglucose positron-emission tomography (FDG-PET)/CT scan. D: FDG-PET/CT scan. Lymph node clustering and metastasis from the right parotid gland to the lateral pharyngeal lymph node (red arrow) and the right supraclavicular lymph node can be seen. E: Pre-pembrolizumab treatment finding. The tumour in the lower right ear was self-destructive.

related adverse event was grade 1 pneumonia on both lungs; therefore, treatment was continued. To date, PR has been maintained after 14 cycles of pembrolizumab.

\section{Discussion}

We report a case of successful treatment of recurrent metastatic parotid cancer with pembrolizumab. Histopathological biopsy analysis at the time of recurrence showed a CPS $>20$; thus, pembrolizumab was indicated.

A search of PubMed for articles on the use of pembrolizumab for recurrent or metastatic parotid cancer yielded a single case of undifferentiated parotid cancer (5).

However, no cases of acinic cell carcinoma of the parotid gland have been reported, which makes this the first of its kind. Acinic cell carcinoma accounts for approximately $10 \%$ of all salivary gland cancer, with $90-95 \%$ occurring in the parotid gland. The average age of patients is 50 years at diagnosis, and the ratio of men to women is $1: 1.5$ (9). It usually progresses slowly and is classified as low-grade cancer. However, there are cases of pulmonary metastasis and high malignant transformation. In 1988, Stanley et al. reported the first case of high-grade transformation of acinic cell carcinoma (10). The 5-year survival rate for patients with acinic cell carcinoma is reported to be $100 \%$ for the
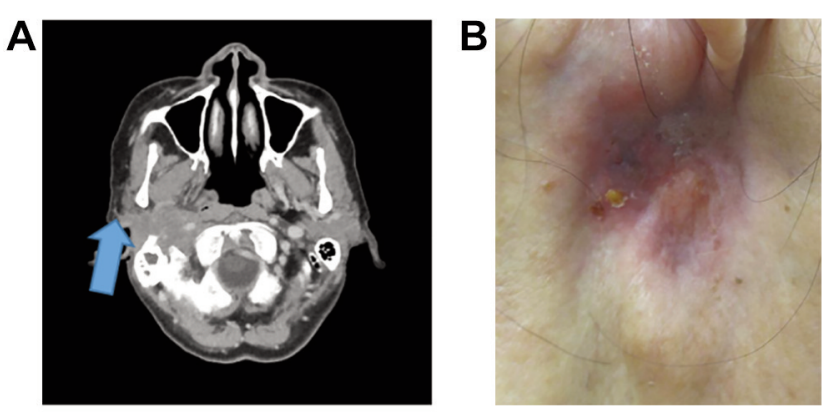

Figure 3. Findings following five cycles of pembrolizumab for acinic cell carcinoma. A: Cervical contrast-enhanced computed tomography scan (axial section). The lesion that was bulging from the skin showed a prominent reduction (blue arrow). B: Findings after treatment showing shrinkage of the right hypodermic tumour.

usual type, $69 \%$ for high-grade transformation, and longterm survival (15-20 years) is approximately $80 \%$ (11). It has also been reported that $15 \%$ of cases relapse within 5 years (12). High-grade transformation is characterised by necrotic and increased mitotic images, high Ki-67 level, perineural and vascular invasion, and infiltrative proliferation (13).

The histopathological specimen in our case at the time of surgery showed mild vascular and skeletal muscle invasion 


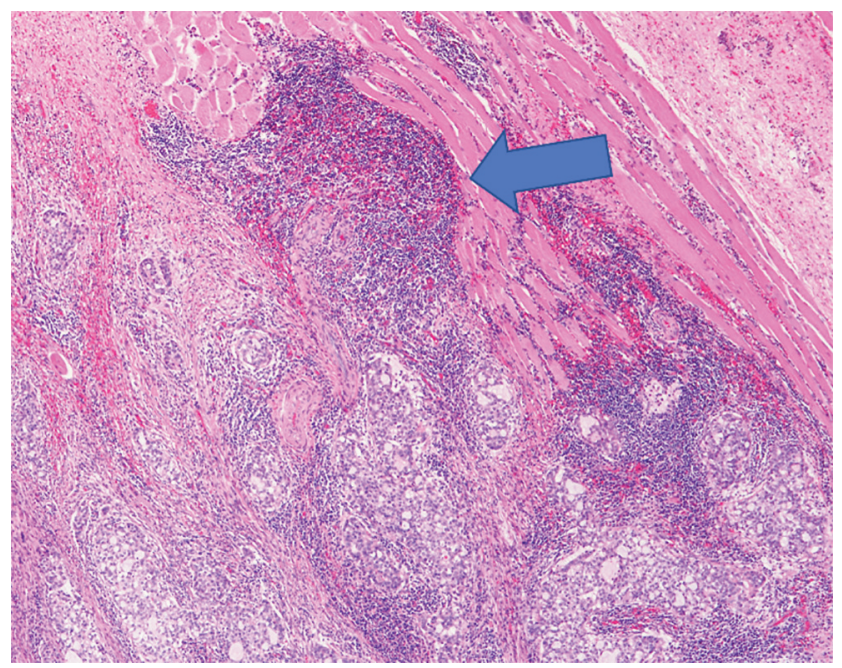

Figure 4. Postoperative pathology of acinic cell carcinoma. Mild vascular invasion and skeletal muscle invasion (blue arrow). Haematoxylin and eosin staining; $\times 40$.

(Figure 4); however, the Ki-67 labelling rate was low (approximately 10\%), and there was no high-grade transformation.

Parotid cancer has a wide variety of histopathological types, and there is a limited number of clinical trials on drug therapy for recurrent and metastatic cases. Cell-killing anticancer drugs with proven antitumoral effects include cisplatin, cyclophosphamide, doxorubicin, paclitaxel, and vincristine $(14,15)$. Phase II trials of these single agents or their combinations have reported overall response rates between 14 and $50 \%$ and a median overall OS between 12.5 and 21 months (16).

On the contrary, KEYNOTE-048 compared the EXTREME regimen of cisplatin plus cetuximab and fluorouracil, the conventional standard of care for recurrent or metastatic head and neck SCC of the oral cavity, pharynx, and larynx with pembrolizumab alone. In the group with a CPS $>1$ and a CPS $>20$, pembrolizumab alone was shown to significantly prolong OS compared to the EXTREME group (4).

Based on these results, for CPS $>1$ and CPS $>20$, the recommended first-line treatment for recurrent or metastatic SCC of the head and neck is pembrolizumab as a single agent as category 1 . Although patients with salivary gland cancer were not included in the KEYNOTE-048 study, pembrolizumab can be administered for salivary gland cancer and with insurance coverage in Japan.

In our patient's case, the CPS was $>20$; therefore, we considered that pembrolizumab alone might be effective. The KEYNOTE-028 study, a phase Ib trial of single-agent pembrolizumab for advanced salivary gland cancer including parotid cancer, showed an overall response rate of $12 \%$ and median OS of 13 months (17).

One patient with acinic cell carcinoma was enrolled in the KEYNOTE-028 trial. As far as we are aware, there are no previous reports on the usefulness of ICIs for treating acinic cell carcinoma. In Japan, pembrolizumab is approved by insurance for the treatment of salivary gland cancer. However, outside Japan, pembrolizumab is not usually administered for salivary adenocarcinoma, which makes this case important. In the future, more case reports on this topic should lead to further clinical trials and new evidence.

\section{Conclusion}

We reported a case of successful pembrolizumab treatment for recurrent or metastatic parotid cancer. After treatment with pembrolizumab, skin and imaging findings showed shrunken lesions and ongoing PR. For recurrent or metastatic salivary gland cancer, pembrolizumab treatment may be successful for patients with CPS score $>20$ and should therefore be considered as a treatment option.

\section{Ethical Statement}

Informed consent was obtained from the patient for treatment, inclusion in medical research, and publication of medical data.

\section{Conflicts of Interest}

All Authors have no conflicts of interest, potential conflicts, or financial relationships to disclose.

\section{Authors' Contributions}

Gai Yamashita drafted the article. Isaku Okamoto designed the study and revised the article. Akira Shimizu, Kunihiko Tokashiki, Takuro Okada, and Hiroki Sato treated the patient. Kiyoaki Tsukahara supervised the study.

\section{Acknowledgements}

The Authors would like to thank Editage (www.editage.com) for English language editing.

\section{References}

1 Laurie SA and Licitra L: Systemic therapy in the palliative management of advanced salivary gland cancers. J Clin Oncol 24(17): 2673-2678, 2006. PMID: 16763282. DOI: 10.1200/ JCO.2005.05.3025

2 El-Naggar AK, Chan JKC, Rubin Grandis J, Takada T and Slootweg PJ: WHO Classification of Head and Neck Tumours. Fourth Edition. International Agency for Research on Cancer, 2017.

3 Pfister DG, Spencer S, Adelstein D, Adkins D, Anzai Y, Brizel DM, Bruce JY, Busse PM, Caudell JJ, Cmelak AJ, Colevas AD, 
Eisele DW, Fenton M, Foote RL, Galloway T, Gillison ML, Haddad RI, Hicks WL, Hitchcock YJ, Jimeno A, Leizman D, Maghami E, Mell LK, Mittal BB, Pinto HA, Ridge JA, Rocco JW, Rodriguez CP, Shah JP, Weber RS, Weinstein G, Witek M, Worden F, Yom SS, Zhen W, Burns JL and Darlow SD: Head and neck cancers, version 2.2020, NCCN clinical practice guidelines in oncology. J Natl Compr Canc Netw 18(7): 873898, 2020. PMID: 32634781. DOI: 10.6004/jnccn.2020.0031

4 Burtness B, Harrington KJ, Greil R, Soulières D, Tahara M, de Castro G Jr, Psyrri A, Basté N, Neupane P, Bratland Å, Fuereder T, Hughes BGM, Mesía R, Ngamphaiboon N, Rordorf T, Wan Ishak WZ, Hong RL, González Mendoza R, Roy A, Zhang Y, Gumuscu B, Cheng JD, Jin F, Rischin D and KEYNOTE-048 Investigators: Pembrolizumab alone or with chemotherapy versus cetuximab with chemotherapy for recurrent or metastatic squamous cell carcinoma of the head and neck (KEYNOTE048): a randomised, open-label, phase 3 study. Lancet 394(10212): 1915-1928, 2019. PMID: 31679945. DOI: 10.1016/S0140-6736(19)32591-7

5 Wiggins A, Arter Z and Kerns T: The three P's: Parotid, PD-L1, and pembrolizumab. Case Rep Oncol Med 2019: 2305315, 2019. PMID: 31308983. DOI: 10.1155/2019/2305315

6 Okamoto I, Sato H and Tsukahara K: Overall survival and PD-L1 expression in patients with recurrent or metastatic head and neck cancer treated with nivolumab. Auris Nasus Larynx 47(4): 676-686, 2020. PMID: 32439271. DOI: 10.1016/j.anl.2020.04.001

7 Sobin LH, Gospodarowicz MK and Wittekind C (eds.): International Union against Cancer. TNM Classification of Malignant Tumours. Hoboken, 2010.

8 Eisenhauer EA, Therasse P, Bogaerts J, Schwartz LH, Sargent D, Ford R, Dancey J, Arbuck S, Gwyther S, Mooney M, Rubinstein L, Shankar L, Dodd L, Kaplan R, Lacombe D and Verweij J: New response evaluation criteria in solid tumours: revised RECIST guideline (version 1.1). Eur J Cancer 45(2): 228-247, 2009. PMID: 19097774. DOI: 10.1016/j.ejca.2008.10.026

9 Stanley RJ, Weiland LH, Olsen KD and Pearson BW: Dedifferentiated acinic cell (acinous) carcinoma of the parotid gland. Otolaryngol Head Neck Surg 98(2): 155-161, 1988. PMID: 3128758. DOI: 10.1177/019459988809800210

10 Federspil PA, Constantinidis J, Karapantzos I, Pahl S, Markmann HU and Iro H: [Acinic cell carcinomas of the parotid gland. A retrospective analysis]. HNO 49(10): 825-830, 2001. PMID: 11699143. DOI: 10.1007/s001060170031
11 Lima RA, Tavares MR, Dias FL, Kligerman J, Nascimento MF, Barbosa MM, Cernea CR, Soares JR, Santos IC and Salviano S: Clinical prognostic factors in malignant parotid gland tumors. Otolaryngol Head Neck Surg 133(5): 702-708, 2005. PMID: 16274796. DOI: 10.1016/j.otohns.2005.08.001

12 Gomez DR, Katabi N, Zhung J, Wolden SL, Zelefsky MJ, Kraus DH, Shah JP, Wong RJ, Ghossein RA and Lee NY: Clinical and pathologic prognostic features in acinic cell carcinoma of the parotid gland. Cancer 115(10): 2128-2137, 2009. PMID: 19309749. DOI: $10.1002 /$ cncr.24259

13 Nagao T: "Dedifferentiation" and high-grade transformation in salivary gland carcinomas. Head Neck Pathol 7 Suppl 1: S37S47, 2013. PMID: 23821210. DOI: 10.1007/s12105-013-0458-8

14 Airoldi M, Pedani F, Succo G, Gabriele AM, Ragona R, Marchionatti $\mathrm{S}$ and Bumma C: Phase II randomized trial comparing vinorelbine versus vinorelbine plus cisplatin in patients with recurrent salivary gland malignancies. Cancer 91(3): 541-547, 2001. PMID: 11169936. DOI: 10.1002/10970142(20010201)91:3<541::aid-cncr1032>3.0.co;2-y

15 Gilbert J, Li Y, Pinto HA, Jennings T, Kies MS, Silverman P and Forastiere AA: Phase II trial of taxol in salivary gland malignancies (E1394): a trial of the Eastern Cooperative Oncology Group. Head Neck 28(3): 197-204, 2006. PMID: 16470745. DOI: $10.1002 /$ hed 20327

16 Haddad R, Colevas AD, Krane JF, Cooper D, Glisson B, Amrein PC, Weeks L, Costello R and Posner M: Herceptin in patients with advanced or metastatic salivary gland carcinomas. A phase II study. Oral Oncol 39(7): 724-727, 2003. PMID: 12907212. DOI: 10.1016/s1368-8375(03)00097-6

17 Cohen RB, Delord JP, Doi T, Piha-Paul SA, Liu SV, Gilbert J, Algazi AP, Damian S, Hong RL, Le Tourneau C, Day D, Varga A, Elez E, Wallmark J, Saraf S, Thanigaimani P, Cheng J and Keam B: Pembrolizumab for the treatment of advanced salivary gland carcinoma: Findings of the phase 1b KEYNOTE-028 study. Am J Clin Oncol 41(11): 1083-1088, 2018. PMID: 29462123. DOI: 10.1097/COC.0000000000000429

Received December 4, 2021

Revised December 16, 2021

Accepted December 18, 2021 